\title{
Nonlinear Excitation Controller for Power Systems using Zero Dynamic Design Approach
}

\author{
M. A. Mahmud, Student Member, IEEE, M. J. Hossain, and H. R. Pota
}

\begin{abstract}
This paper presents a nonlinear excitation controller design technique for interconnected power systems. A zero dynamic design approach is used to design the controller which is useful when the system is partially linearizable and the obtained control law, using this approach is simpler as compared to that of a fully or exactly linearizable system. The performance of this controller is tested on a single machine infinite bus (SMIB) system over a wide variation of operating region and compared to the nonlinear excitation controller which is designed using the concept of exact linearization. Simulation results show that the proposed control scheme can improve the stability of power systems, regardless of operating points and the performance is very similar to that of exactly linearized controller.
\end{abstract}

\section{INTRODUCTION}

Control of modern electric power systems becomes more and more challenging as the present trends of power system engineers are to operate the power systems closer to their stability limits. The stability of power system is very essential for secure system operation because an unsecured system can undergo non-periodic major cascading disturbances, or blackouts, which have serious consequences. The world power grids are experiencing many blackouts in recent years [1] which can be attributed to special causes, such as equipment failure, overload, lightning stores, or unusual operating conditions.

The stable operation of power systems can be ensured by using high-performance controllers which regulate the system under diversity of operating conditions. The fundamental phenomenon of secure power system operation is investigated in [2] which has explored a variety of machine loading, machine inertias, and system external impedances with a determination of the oscillation and damping characteristics of voltage or speed following a small disturbance in mechanical torque. Based on this phenomenon, many techniques to assess the stability of the power system have been proposed. In [3], there is an extensive description of power system stabilizers (PSSs) which are now widely used in industries. Some improved methodologies of PSS design are proposed in [4], [5] which has large disturbance rejection capacity. Recently, a coordinated PSS design approach is proposed in [6]. Also in our recent work [7], [8], [9], the stability of power systems is analyzed with the variation of dynamic loads and the factors related to the power systems stability are investigated. In all of these works [3-9], the stability of power systems is studied

M. A. Mahmud and H. R. Pota are with the School of Engineering and Information Technology (SEIT), The University of New South Wales at Australian Defence Force Academy (UNSW@ADFA), Canberra, ACT 2600, Australia. E-mail: Md.Mahmud@student.adfa.edu.au and h.pota@adfa.edu.au.

M. J. Hossain is with the School of Information Technology and Electrical Engineering (ITEE), University of Queensland, St Lucia, Brisbane, QLD 4072, Australia. E-mail: m.hossain9@uq.edu.au. based on the linearized model which give the idea about the stability under a fixed set of operating points.

It is well known that application of nonlinear control theory can provide better operation with respect to the variation of operating points by canceling the inherent systems nonlinearities. Feedback linearization control schemes are widely used in power system to design controllers. It deals with the techniques for transforming original system models into equivalent model with a simpler form. The main idea of this approach is to algebraically transform nonlinear system dynamics into a (fully or partly) linear one, so that the linear control techniques can be applied. When the relative degree of a system is equal to the order of the system, then the system is fully linearized but when the relative degree is less than the order of the system, the system is partially linearized. A simple form of feedback linearization called direct feedback linearization is proposed in [10], [11] to design controller for single machine infinite bus system as well as for multimachine power systems and the controller is independent of operating points. In direct feedback linearization approach, the system is transformed into another form by introducing a new state but the main concept of feedback linearization is not used. The performance of nonlinear excitation controller for power system is investigated in [12], [13], [14] where the main concept of feedback linearization is used and the system is considered as fully linearizable.

In the design of nonlinear controller, the relative degree which depends on the output function of the system is the main key. In order to design feedback linearizable controller, different approaches need to be used based on the relative degree of the system. If the relative degree of the system is equal to the order of the system, the exact linearization approach of feedback linearization needs to be used. On the other hand, if the relative degree of the system is less than the order of the system, the zero dynamic design approach of feedback linearization needs to be used.

The aim of this paper is to present the zero dynamic design approach, to design a excitation controller for power system. This paper also presents the detailed overview of zero dynamic design approach. The designed controller is implemented on a SMIB system. The performance of the designed controller is evaluated over a wide variation of operating points. Finally, the performance of this controller is compared to that of the fully linearizable one.

The rest of the paper is organized as follows. In Section II, the mathematical modeling of a single machine infinite bus system is given. The detailed about zero dynamic design approach is given in Section III. Section IV shows the nonlinear excitation controller design for power system using zero 


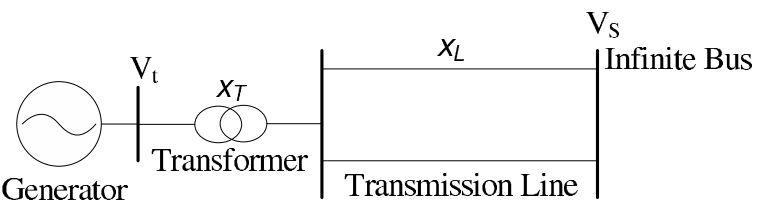

Fig. 1. Test system

dynamic design approach. Simulation results including the comparison of performances are presented in V. Finally, the paper is concluded with future trends and further recommendation in Section VI.

\section{Power SYSTEM MODEL}

Power systems can be modeled at several different levels of complexities, depending on the intended application of the model. Fig. 1 shows a single machine infinite bus system which is the focus of this paper. Since SMIB system qualitatively exhibits the important aspects of the behavior of a multimachine system and is relatively simple to study, it is extremely useful in studying general concepts of power system stability [10].

With some typical assumptions, the classical third-order dynamical model of a SMIB power system as shown in Fig. 1 can be modeled by the following set of differential equations [3], [13]:

Generator mechanical dynamics:

$$
\begin{aligned}
\dot{\delta} & =\omega \\
\dot{\omega} & =-\frac{D}{2 H} \omega+\frac{1}{2 H}\left(P_{m}-P_{e}\right)
\end{aligned}
$$

where $\delta$ is the power angle of the generator, $\omega$ is the rotor speed with respect to synchronous reference, $H$ is the inertia constant of the generator, $P_{m}$ is the mechanical input power to the generator which is assumed to be constant, $D$ is the damping constant of the generator, and $P_{e}$ is the active electrical power delivered by the generator.

Generator electrical dynamics:

$$
\dot{E}_{q}^{\prime}=\frac{1}{T_{d o}}\left(E_{f}-E_{q}\right)
$$

where $E_{q}^{\prime}$ is the quadrature-axis transient voltage of the generator, $E_{q}$ is the quadrature-axis voltage of the generator, $T_{d o}$ is the direct-axis open-circuit transient time constant of the generator, and $E_{f}$ is the equivalent voltage in the excitation coil.
Electrical equations:

$$
\begin{aligned}
E_{q} & =\frac{x_{d \Sigma}}{x_{d \Sigma}^{\prime}} E_{q}^{\prime}-\left(x_{d}-x_{d}^{\prime}\right) \frac{V_{s}}{x_{d \Sigma}^{\prime}} \cos \delta \\
I_{q} & =\frac{V_{s}}{x_{d \Sigma}^{\prime}} \sin \delta \\
I_{d} & =\frac{E_{q}^{\prime}}{x_{d \Sigma}^{\prime}}-\frac{V_{s}}{x_{d \Sigma}^{\prime}} \cos \delta \\
P_{e} & =\frac{V_{s} E_{q}^{\prime}}{x_{d \Sigma}^{\prime}} \sin \delta \\
Q_{e} & =\frac{V_{s} E_{q}^{\prime}}{x_{d \Sigma}^{\prime}} \cos \delta-\frac{V_{s}^{2}}{x_{d \Sigma}} \\
V_{t} & =\sqrt{\left(E_{q}^{\prime}-X_{d}^{\prime} I_{d}\right)^{2}+\left(X_{d}^{\prime} I_{q}\right)^{2}}
\end{aligned}
$$

where $x_{d \Sigma}=x_{d}+x_{T}+x_{L}, x_{d \Sigma}^{\prime}=x_{d}^{\prime}+x_{T}+x_{L}, x_{d}$ is the direct-axis synchronous reactance, $x_{d}^{\prime}$ is the direct axis transient reactance, $x_{T}$ is the reactance of the transformer, $x_{L}$ is the reactance of the transmission line, $I_{d}$ and $I_{q}$ are direct and quadrature axis currents of the generator respectively, $V_{s}$ is the infinite bus voltage, $Q_{e}$ is the generator reactive power delivered to the infinite bus, and $V_{t}$ is the terminal voltage of the generator.

Using the electrical equations into the mechanical and electrical dynamics equation (1)-(3) of the system, the complete mathematical model of SMIB system can be written as follows:

$$
\begin{aligned}
\dot{\delta} & =\omega \\
\dot{\omega} & =-\frac{D}{2 H} \omega+\frac{P_{m}}{2 H}-\frac{1}{2 H} \frac{V_{s} E_{q}^{\prime}}{x_{d \Sigma}^{\prime}} \sin \delta \\
\dot{E}_{q}^{\prime} & =-\frac{1}{T_{d}^{\prime}} E_{q}^{\prime}+\frac{1}{T_{d o}} \frac{x_{d}-x_{d}^{\prime}}{x_{d \Sigma}^{\prime}} V_{s} \cos \delta+\frac{1}{T_{d o}} E_{f}
\end{aligned}
$$

where $T_{d}^{\prime}=\frac{x_{d \Sigma}^{\prime}}{x_{d \Sigma}} T_{d o}$ is the time constant of the field winding. The numerical values of the system parameters are given in Appendix A.

\section{Zero Dynamic Design ApProach}

The control obtained by using exact linearization approach is quite complicated which can be seen in our previous work [12], [14]. In this section, the zero dynamic design approach will be discussed which does not need to exactly linearize all system's state equations, but just a part of them.

The dynamic behavior of any systems can be classified into external dynamic and internal dynamics. Of these, the main concern is the external dynamic of the system which needs to be stable as well as it should have good performance. On the other hand, the internal dynamics of the systems need to be stable only. This is the basic idea of zero dynamic design approach which will be clarified in the following discussions.

Let the nonlinear system be written in the form:

$$
\begin{aligned}
& \dot{x}(t)=f(x)+g(x) u \\
& y(t)=h(x)
\end{aligned}
$$


where $x \in \mathbf{R}^{n}$ is the state vector; $u \in \mathbf{R}$ is the control vector; $y \in \mathbf{R}$ is the output vector; $f(x)$ and $g(x)$ are the $n$ dimensional vector fields in the state space; $h(x)$ is the scalar function of $x$.

Before going inside the zero dynamic design approach, it is essential to define the Lie derivative and relative degree which are given below:

\section{Definition 1: (Lie Derivative)}

For a given differentiable scalar function $h(x)$ of $x=$ $\left[\begin{array}{lllll}x_{1} & x_{2} & x_{3} & \cdots & x_{n}\end{array}\right]^{T}$ and a vector field $f(x)=$ $\left[\begin{array}{lllll}f_{1} & f_{2} & f_{3} & \cdots & f_{n}\end{array}\right]^{T}$, the new scalar function, denoted by $L_{f} h(x)$, is obtained by the following operation

$$
L_{f} h(x)=\frac{\partial h(x)}{\partial x} f(x)=\sum_{i=1}^{n} \frac{\partial h(x)}{\partial x_{i}} f_{i}(x)
$$

which is called Lie derivative of function $h(x)$ along the vector field $f(x)$.

Definition 2: (Relative Degree)

If the Lie derivative of the function $L_{f}^{r-1} h(x)$ along vector field $g(x)$ is not equal to zero in a neighborhood $\Omega$, i.e.,

$$
L_{g} L_{f}^{r-1} h(x) \neq 0
$$

then it can be said that the system have relative degree $r$ in $\Omega$.

The relative degree of a system depends on the selection of output of the system. In nonlinear control theory, the relative degree of the system is very important. The design procedure of a nonlinear controller will be different based on the relative degree. If the relative degree of a system is equal to the order ( $n$ ) of the system, then it can be said that the system is fully linearizable, or exactly linearizable but $r<n$, the system is partially linearized. The zero dynamic design approach is applicable on the second case.

To implement zero dynamic design approach, let $r \leq n$. Under this condition, for nonlinear system (7), the following expressions are true.

$$
\begin{gathered}
L_{g} L_{f}^{1-1} h(x)=L_{g} L_{f}^{2-1} h(x)=\cdots=L_{g} L_{f}^{n-2} h(x)=0 \\
L_{g} L_{f}^{r-1} h(x) \neq 0
\end{gathered}
$$

Now the mapping from $x$ space to $z$ space is constructed through nonlinear coordinate transformation by choosing

$$
z_{1}=y=h(x)=L_{f}^{1-1} h(x)
$$

then we can write

$$
\dot{z}_{1}=\frac{\partial h(x)}{\partial x} \dot{x}
$$

Substituting equation (7) into the above equation for $\dot{x}$, we get $\dot{z}_{1}=\frac{\partial h(x)}{\partial x} f(x)+\frac{\partial h(x)}{\partial x} g(x) u=L_{f}^{2-1} h(x)+L_{g} L_{f}^{1-1} h(x) u$

As $L_{g} L_{f}^{1-1} h(x)=0$, from the above equation it can be written that

$$
\dot{z}_{1}=L_{f} h(x)=z_{2}
$$

In a similar way

$$
\begin{aligned}
\dot{z}_{2}=L_{f}^{2} h(x) & =z_{3} \\
\vdots & \\
\vdots & \\
\dot{z}_{r-1}=L_{f}^{n-1} h(x) & =z_{n}
\end{aligned}
$$

Since $L_{g} L_{f}^{r-1} h(x) \neq 0$, finally we can write

$$
\dot{z}_{r}=L_{f}^{r} h(x)+L_{g} L_{f}^{r-1} h(x) u
$$

Here, the first $r$ equation is linearized. Now, let the rest $(n-r)$ equations are

$$
\begin{aligned}
\dot{z}_{r+1}= & L_{f}^{r+1} \varphi(x) \\
\dot{z}_{r+2}= & L_{f}^{r+2} \varphi(x) \\
& \vdots \\
\dot{z}_{n}= & L_{f}^{n} \varphi(x)
\end{aligned}
$$

which should be selected in such a way that they must satisfy

$$
L_{g} \varphi_{i}(x)=0 \quad r+1 \leq i \leq n
$$

and the Jacobian Matrix at $x=x_{0}$

$$
J_{\varphi}=\left.\frac{\partial \varphi(x)}{\partial x}\right|_{x=x_{0}}
$$

is nonsingular. Finally, the transformed system can be written as

$$
\begin{array}{rlll}
\dot{z}_{1} & & = & z_{2} \\
\dot{z}_{2} & & = & z_{3} \\
& \ldots & \ldots & \\
\dot{z}_{r-1} & & = & z_{r} \\
\dot{z}_{r} & = & L_{f}^{r} h\left(\phi^{-1}(z)\right)+L_{g} L_{f}^{r-1} h\left(\phi^{-1}(z)\right) u \\
\dot{z}_{r+1} & & & L_{f} \varphi_{r+1}\left(\phi^{-1}(z)\right) \\
& \ldots & \ldots & \\
\dot{z}_{n} & & & L_{f} \varphi_{n}\left(\phi^{-1}(z)\right)
\end{array}
$$

Here, $x=\phi^{-1}(z)$ and

$$
\phi(x)=\left[\begin{array}{c}
z_{1}(x) \\
\vdots \\
z_{r}(x) \\
z_{r+1}(x) \\
\vdots \\
z_{n}(x)
\end{array}\right]=\left[\begin{array}{c}
h(x) \\
\vdots \\
L_{f}^{r-1} h(x) \\
\varphi_{r+1}(x) \\
\vdots \\
\varphi_{n}(x)
\end{array}\right]
$$

For the sake of simplicity, we introduce the following notations:

$$
\begin{aligned}
\zeta & =\left[\begin{array}{lllll}
z_{1} & z_{2} & z_{3} & \cdots & z_{r}
\end{array}\right]^{T} \\
\eta & =\left[\begin{array}{lllll}
z_{r+1} & z_{r+2} & z_{r+3} & \cdots & z_{n}
\end{array}\right]^{T}
\end{aligned}
$$


Therefore, using the above mentioned notations we can write

$$
\begin{array}{rlll}
\dot{z}_{1} & & & z_{2} \\
\dot{z}_{2} & & = & z_{3} \\
& \ldots \ldots & \\
\dot{z}_{r-1} & = & z_{r} \\
\dot{z}_{r} & = & a(\zeta, \eta)+b(\zeta, \eta) u \\
\dot{\eta} & = & q(\zeta, \eta)
\end{array}
$$

where

$$
\begin{aligned}
& a(\zeta, \eta)=\left.L_{f}^{r} h(x)\right|_{x=\phi^{-1}(\zeta, \eta)}=L_{f}^{r} h\left(\phi^{-1}(\zeta, \eta)\right) \\
& b(\zeta, \eta)=\left.L_{g} L_{f}^{r-} h(x)\right|_{x=\phi^{-1}(\zeta, \eta)}=L_{g} L_{f}^{r-1} h\left(\phi^{-1}(\zeta, \eta)\right)
\end{aligned}
$$

and

$$
q(\zeta, \eta)=\left[\begin{array}{c}
q_{r+1}(\zeta, \eta) \\
q_{r+2}(\zeta, \eta) \\
\vdots \\
q_{n}(\zeta, \eta)
\end{array}\right]=\left[\begin{array}{c}
L_{f} \phi_{r+1}\left(\phi^{-1}(\zeta, \eta)\right) \\
L_{f} \phi_{r+2}\left(\phi^{-1}(\zeta, \eta)\right) \\
\vdots \\
L_{f} \phi_{n}\left(\phi^{-1}(\zeta, \eta)\right)
\end{array}\right]
$$

In general, the output function is chosen in such a way that the output equation $y=h(x)=0$ at $x=x_{0}$. Therefore, the output $y=h(x)$ is the actually dynamic deviation of the practical output (dynamic response) from the output at an equilibrium point. Now, if we use the control means to impose on that dynamic deviation of the output of the system keeps zero at any time, i.e.,

$$
y=h(x)=0 \quad 0 \leq t \leq \infty
$$

then from external dynamics of the control system we can consider that the system is so stable that under the influence of any disturbance as the output of the system does not change.

Since $y=h(x)=Z_{1}$ has been set to zero at any time, therefore

$$
\dot{z}_{1}=0
$$

Similarly, the first $r$ components of coordinates $z$ are

$$
\zeta=\left[\begin{array}{lllll}
z_{1} & z_{2} & z_{3} & \cdots & z_{r}
\end{array}\right]^{T}=0
$$

for all $t \geq 0$ and there exists

$$
\dot{z}_{r}=0
$$

Finally, the first $r$ equations will vanish which implies that

$$
\dot{\eta}=q(0, \eta)
$$

Since external dynamics of the system equals zero under the effect of the control strategy, the above differential equation set describes actually the internal dynamics of the system. Those equations which determine internal dynamics of the system are called the zero dynamics equations of the original system. If the zero dynamics of system is stable, then the whole system must be stable and the output variable $y(t)$ will keep constant under any disturbance. Therefore, it is essential to check the stability of zero dynamics of the system before designing the controller.
If the zero dynamics of a nonlinear system is stable, then the control law can be obtained by using equation (9) and (10). Thus, from equation (9) and (10),

$$
L_{f}^{r} h(x)+L_{g} L_{f}^{r-1} h(x) u=0
$$

which implies the following control law

$$
u=-\frac{L_{f}^{r} h(x)}{L_{g} L_{f}^{r-1} h(x)}
$$

This concept of zero dynamic design approach is applied to a power system model in the following section.

\section{Nonlinear Excitation CONTROLler For Power SYSTEMS}

The power system model represented by equation (4)-(6) can be written in the form of equation (7)-(8) where

$$
x=\left[\begin{array}{lll}
\delta & \omega & E_{q}^{\prime}
\end{array}\right]^{T}
$$

$$
\begin{gathered}
f(x)=\left[\begin{array}{c}
\omega \\
-\frac{D}{2 H} \omega+\frac{P_{m}}{2 H}-\frac{1}{2 H} \frac{V_{s} E_{q}^{\prime}}{x_{d \Sigma}^{\prime}} \sin \delta \\
-\frac{1}{T_{d}^{\prime}} E_{q}^{\prime}+\frac{1}{T_{d o}} \frac{x_{d}-x_{d}^{\prime}}{x_{d \Sigma}^{\prime}} V_{s} \cos \delta
\end{array}\right] \\
g(x)=\left[\begin{array}{lll}
0 & 0 & \frac{1}{T_{d o}}
\end{array}\right]^{T} \\
u=E_{f}
\end{gathered}
$$

and

$$
y=h(x)=\omega
$$

First, the relative degree of the considered power system model needs to be calculated. With the chosen output function $y=\omega$, the relative degree of power system can be calculated as follows:

$$
L_{f}^{1-1} h(x)=h(x)=\omega
$$

Therefore,

$$
L_{g} L_{f}^{1-1} h(x)=L_{g} h(x)=\frac{\partial h(x)}{\partial x} g(x)=0
$$

Again,

$L_{f}^{2-1} h(x)=L_{f} h(x)=-\frac{D}{2 H} \omega+\frac{P_{m}}{2 H}-\frac{1}{2 H} \frac{V_{s} E_{q}^{\prime}}{x_{d \Sigma}^{\prime}} \sin \delta$

Hence,

$L_{g} L_{f}^{2-1} h(x)=\frac{\partial\left(L_{f} h(x)\right)}{\partial x} g(x)=\frac{1}{2 H} \frac{V_{s}}{x_{d \Sigma}^{\prime}} \sin \delta \frac{1}{T_{d o}} \neq 0$

Therefore, the relative degree of the system is 2 which is less than the order of the system.

Since the relative degree of power system is less than the order of the system, the zero dynamic design approach for designing nonlinear controller can be implemented easily as it is well proven that power system is inherently stable. 


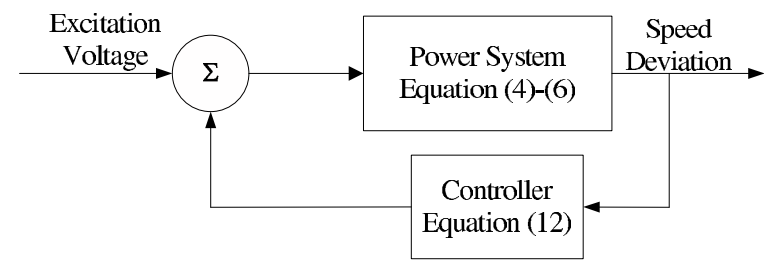

Fig. 2. Excitation controller for power system

To obtain the control law, we need to calculate $L_{f}^{2} h(x)$ which is

$$
\begin{aligned}
L_{f}^{2} h(x) & =-\frac{\omega}{2 H} \frac{V_{s} E_{q}^{\prime}}{x_{d \Sigma}^{\prime}} \cos \delta-\frac{D}{2 H} \dot{\omega} \\
& +\frac{1}{2 H} \frac{1}{T_{d}^{\prime}}\left(\frac{V_{s} E_{q}^{\prime}}{x_{d \Sigma}^{\prime}} \sin \delta-\frac{V_{s}^{2}}{x_{d \Sigma}^{\prime}} \sin \delta\right)
\end{aligned}
$$

Therefore, by using (11) and after doing some simplification, the control can be written as

$$
u=E_{q}-T_{d}^{\prime} \frac{E_{q}}{P_{e}}\left(Q_{e}+\frac{V_{s}^{2}}{x_{d \Sigma}^{\prime}}\right) \Delta \omega-D \frac{E_{q}}{P_{e}} \Delta \dot{\omega}
$$

Here, $E_{q}=x_{a d} I_{f}$ where $x_{a d}$ is the mutual reactance between excitation coil and stator coil and $I_{f}$ is is the per unit excitation current with the no-load rated excitation current as the base value.

Equation (12) represents the control input for the considered power system which is expressed in terms of all measured variables and can be implemented through the excitation of SMIB system. Moreover, the control law is much simpler as compared to that which is obtained from exact linearization [12], [14]. The block diagram representation of power system with this controller is shown in Fig. 2. The performance of this controller is shown in the following section.

\section{Simulation Results}

In this section, the dynamical response of SMIB as shown in Fig. 1, under different operating conditions is tested using the proposed control scheme. The performance of this proposed nonlinear excitation controller is also compared with other controller [14] which is designed by using exact feedback linearization. The simulations are carried out under two different operating points.

In the first simulation, the initial value of the generator rotor angle $\delta(0)=24^{\circ}$ which corresponds to the other initial values of the state variables. The resulting speed deviation of the controlled system is shown in Fig. 3. In Fig. 3, the solid line represents the performance of nonlinear excitation controller which is designed by using exact linearization approach and the dashed line presents the response of the proposed nonlinear excitation controller that is designed through zero dynamic design approach. From Fig. 3 it is also clear that the both controllers perform in a similar manner.

In the second simulation, the initial value of the generator rotor angle is considered as $\delta(0)=72^{\circ}$ and the corresponding initial values of the other states are also taken into account. Under this condition the performances of exact linearizing controller and proposed controller are shown in Fig. 4. In

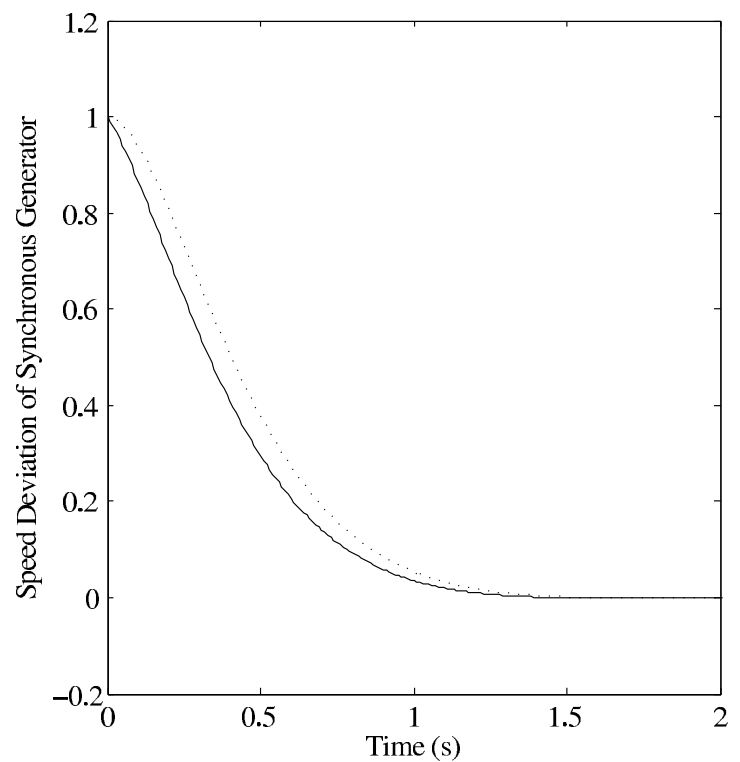

Fig. 3. Speed deviation of synchronous generator when $\delta(0)=24^{\circ}$ (The solid line represent the speed deviation for exact linearizing excitation controller, whereas the dashed line represents the same for zero dynamic design approach)

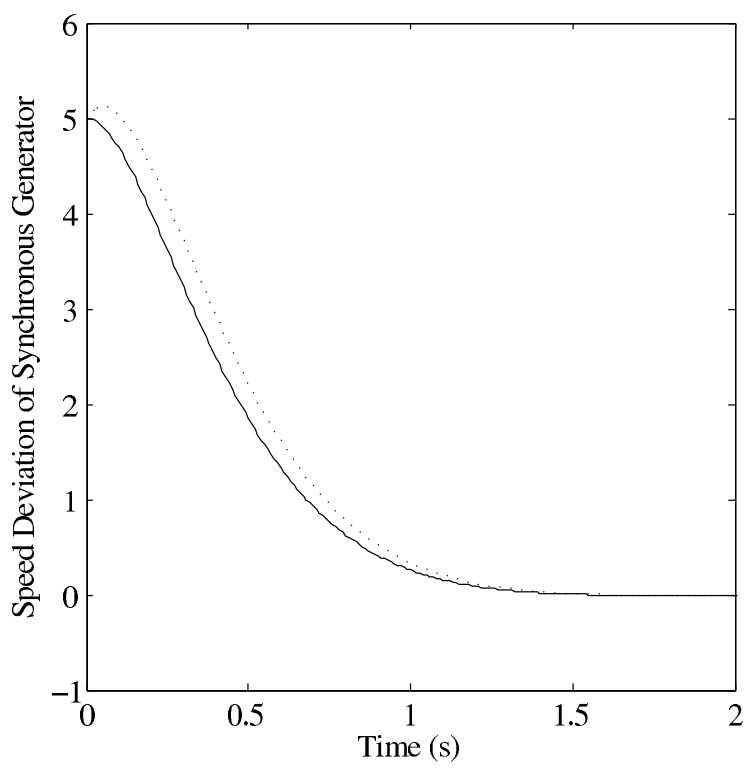

Fig. 4. Speed deviation of synchronous generator when $\delta(0)=72^{\circ}$ (The solid line represent the speed deviation for exact linearizing excitation controller, whereas the dashed line represents the same for zero dynamic design approach)

Fig. 4, the solid line shows the speed deviation of synchronous generator when an exact linearizing excitation controller is used. On the other hand, the dashed line represents the performances of nonlinear excitation controller which is designed by using zero dynamic deign approach and this performance is very close to that of exact linearizing controller.

Simulation results clearly show that nearly same performance can be obtained by using a simple control law in both cases of simulation. The complexity and computation burden is less in zero dynamic design approach as compared to the exact linearization approach of nonlinear controller design. 


\section{CONCLUSION}

This paper presents the zero dynamic design approach to design nonlinear excitation controller of power system. The dynamics of the system is classified into external and internal dynamics, to design nonlinear controller using zero dynamic design approach. The internal dynamics of power systems are not the main concerns in the design of this type of controller for power systems because power systems are internally stable system. The control law obtained by this method is simple and practical as compared to that of obtained by exact linearization approach. The performance of this controller is tested and compared with the performance of exact linearizing controller through some simulations. Simulations results clearly represent that there are not far differences in the performance. The future work with deal with the design of such type of controller for power system with dynamic loads. Future work will also deal with the implementation of this controller to handle large power systems models.

\section{REFERENCES}

[1] X. Zhao, X. Zhang, and B. He, "Study on self organized criticality of china power grid blackouts," Energy Conversion and Management, Elsevier, vol. 50, no. 3, pp. 658-661, March 2009.

[2] F. P. Demello and C. Concordia, "Concepts of synchronous machine stability as affected by excitation control," IEEE Trans. on Power Appartus and Systems, vol. PAS-88, no. 4, pp. 316-329, 1969.

[3] P. Kundur, Power System Stability and Control. New York: McGrawHill, 1994.

[4] R. A. Ramos, A. C. P. Martins, and N. G. Bretas, "An improved methodolgy for the design of power system damping controllers," IEEE Trans. on Power Systems, vol. 20, no. 4, pp. 1938-1945, 2005.

[5] G. Gurrala and I. Sen, "Power system stabilizers design for interconnected power systems," IEEE Trans. on Power Systems, vol. 25, no. 2, pp. 1042-1051, 2010.

[6] A. Dysko, W. E. Leithead, and J. O'Reilly, "Enhanched power system stability by coordinated PSS design," IEEE Trans. on Power Systems, vol. 25, no. 1, pp. 413-422, 2010.

[7] M. A. Mahmud, M. J. Hossain, and H. R. Pota, "Investigation of critical parameters for power system stability with dynamic loads," In Proc. of 2010 IEEE PES General Meeting, 2010.

[8] M. J. Hossain, H. R. Pota, V. Ugrinovski, and R. A. Rramos, "Voltage mode stabilization in power systems with dynamic loads," Int. J. of Electric Power and Energy Systems, vol. 32, no. 8, pp. 911-920, 2010.

[9] M. A. Mahmud, M. J. Hossain, and H. R. Pota, "Effect of large dynamic loads on interconnected power systems with power oscillation damping controller (PODC)," In Proc. of 20th Australasian Universities Power Engineering Conference, 2010.

[10] G. Guo, D. J. Hill, and Y. Wang, "Global transient stability and voltage regulation for power systems," IEEE Trans. on Power Systems, vol. 16, no. 4, pp. 678-688, 2001.

[11] — "Nonlinear output stabilization control for multimachine power systems," IEEE Tans. on Circuit and Systems- Part 1, vol. 47, no. 1, pp. 46-52, 2000.

[12] M. A. Mahmud, M. J. Hossain, and H. R. Pota, "Nonlinear excitation control of power systems with dynamic loads via feedback linearization," In Proc. of 20th Australasian Universities Power Engineering Conference, 2010.

[13] Q. Lu, Y. Sun, and S. Mei, Nonlinear Control Systems and Power System Dynamics. Boston/Dordrecht/London: Kluwer Academic Publishers, 2001.

[14] M. A. Mahmud, M. J. Hossain, and H. R. Pota, "Nonlinear excitation control of power systems with dynamic loads via feedback linearization," In Proc. of 18th IFAC World Congress, 2011.

\section{APPENDIX}

The parameters used for the SMIB system are given below: Synchronous generator parameters: $x_{d}=1.863 p u, x_{a d}=1.712 p u, x_{d}^{\prime}=0.257 p u, H=8 s$, $T_{d o}=6.9 s, D=5, \omega_{0}=314.159$.

Transformer Parameter: $x_{T}=0.127 \mathrm{pu}$.

Transmission Line Parameters: $x_{L}=0.4853 \mathrm{pu}$.

Infinite bus voltage, $V_{s}=1.00 \mathrm{pu}$.

Mechanical Power Input, $P_{m}=0.9 \mathrm{pu}$. 